\title{
Preoperative nutrition and postoperative liver function: a correlation study of pediatric living donor liver transplantation ${ }^{\dagger}$
}

Original article

Ye-Feng Lua, Yan Wanga, Ming-Zhu Huanga ${ }^{a}$ Xue-Fei Renª, Lei-Qing Gao , Dan Li ${ }^{b}$, Yan-Fen Lic, Yan Yang ${ }^{\mathrm{d}, *}$

aDivision of Hepatic Surgery, Renji Hospital, School of Medicine, Shanghai Jiao Tong University, Shanghai 200127, China

${ }^{b}$ Division of No. 1 Surgery, Hunan Children's Hospital, Changsha, Hunan 410007, China

'Division of Day Surgery, Renji Hospital, School of Medicine, Shanghai Jiao Tong University, Shanghai 200127, China

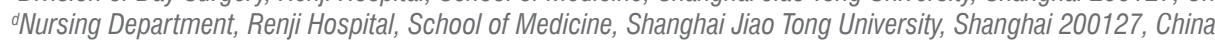

Received: 12 September 2017; Accepted: 18 December 2017; Published: 20 June 2018

\begin{abstract}
Objective: There is little information focusing on the nutritional issue of pediatric recipients before they receive living donor liver transplantation. This study illustrates the relationship between nutritional status and graft liver function and provides a reference regarding nutritional interventions in future studies.

Methods: We prospectively collected data from 30 pediatric living donor liver transplant recipients from January 1, 2016, to June 30 2016. The information included demographic data, preoperative nutritional assessment, and postoperative laboratory examinations. The nutritional assessment included the serum concentration of vitamin $\mathrm{D}$, bone density, trace element, and weight $Z$ value. The laboratory examinations included white blood cell count, neutrophil percentage, hemoglobin, blood platelet, total protein, albumin, total bilirubin, direct bilirubin, alanine transaminase, aspartate aminotransferase (AST), alkaline phosphatase, gamma-glutamyl transpeptidase, creatinine, bile acid, blood glucose (Glu), prothrombin time, international normalized ratio, tacrolimus concentration, and graft-to-recipient weight ratio (GRWR). The data were collected on Days 1, 2, 3, 4, 5, 6, 7, 14, 30, and 60 after liver transplantation. Results: The recipients consisted of $15(50 \%)$ males and 15 (50\%) females. The median age was 7 months (4-48 months). The mean height and weight were $69.07 \pm 9.98 \mathrm{~cm}$ and $8.09 \pm 2.63 \mathrm{~kg}$, respectively. According to the univariate analysis, the gender, diagnosis, blood type, and GRWR did not significantly impact the liver function after the operation. The posttransplantation AST levels and Glu showed significant differences in terms of the nutritional status, with $P<0.05$. The multivariate correlation analysis showed that the serum concentrations of vitamin D and AST were midrange positively correlated, with $P<0.05$.

Conclusions: The nutritional status of patients with biliary atresia is relatively poor. There is a definite midrange positive correlation between nutrition and graft liver function that might play a relatively important role in the recovery of the graft.
\end{abstract}

Keywords: pediatric • liver transplantation • nutrition • correlation

(c) Shanxi Medical Periodical Press.

\section{Introduction}

A variety of primary diseases has led Chinese pediatric patients to suffer from liver cirrhosis, especially biliary atresia (BA), which has threatened their health and ${ }^{\dagger}$ This project was supported by Shanghai Municipal Education
Commission - Gaoyuan Nursing Grant Support (No. Hlgy15004yjx).

*E-mail: yangyan@renji.com lifespan; fortunately, significant progress in the field of liver transplantation ${ }^{1}$ has made this treatment the most effective therapeutic strategy for pediatric patients with end-stage liver diseases. ${ }^{2}$

Several transplantation centers have begun to perform living donor liver transplantation (LDLT) since the first case of pediatric liver transplantation was successfully performed in the mainland of China in May 1996 . $^{3}$ 
The outcomes of pediatric LDLT have been greatly improved and broadly recognized. ${ }^{3}$

Chronic liver disease (CLD) noticeably impairs nutritional status, and malnourishment is almost universally present in patients with end-stage liver disease who undergo liver transplantation. ${ }^{4}$ The importance of assessing the nutritional status during the workup of patients who are candidates for liver replacement is widely recognized. Cirrhotic patients with depleted lean body mass and fat deposits have an increased surgical risk; malnutrition might further impact morbidity, mortality, and costs in the posttransplantation setting. Few studies have examined the modifications in body composition that occur in liver recipients. Depletions in muscle compartment and fat mass have been shown to contribute to malnutrition in cirrhotic patients. Muscle wasting, which is accompanied by reduced muscle function, is likely the most relevant feature in these patients.

However, little attention has been paid to the relationship between the preoperational nutritional status and postoperational liver function in the pediatric liver transplant population. This prospective study aims to summarize the 30 cases of pediatric recipients who received liver transplants in the first half of 2016 and to provide a reference for future clinical practice.

\section{Methods}

This was a single-center prospective survey conducted at the Department of Hepatic Surgery, Renji Hospital, School of Medicine, Shanghai Jiaotong University, China, which was conducted after approval by the institutional review board (IRB). The follow-up period was 60 days, and a total of 30 pediatric liver transplantation patients from January 1, 2016, to June 30, 2016, were recruited. Patients with missing data and those who did not complete the follow-up were excluded.

\subsection{Preoperative nutritional evaluation}

Pretransplant nutritional assessment was performed in the Shanghai Children's Medical Center. The liver function was evaluated in the Renji Hospital. A specialized nurse was appointed to oversee the collection of the data and to coordinate for any other issues.

\subsection{Postoperative management}

We began to monitor and record the laboratory data and analyze the imaging examinations of the pediatric recipients when they stayed in the ICU. These data included white blood cell (WBC), hemoglobin (HB), blood platelet (PLT), serum total bilirubin (TB), albumin (ALB), alanine transaminase (ALT), aspartate aminotransferase (AST), prothrombin time (PT), international normalized ratio and tacrolimus concentration. The therapy was adjusted according to the results of the examinations to help the children live through the early phase after transplantation.

\subsection{Statistical analysis}

Continuous variables were presented as the mean ( \pm standard deviation) and median (range), and categorical variables were represented as frequencies. SPSS (version 16.0; SPSS Inc., Chicago, IL, USA) was used for the analysis. Statistical inference and correlation analyses were adopted. $P<0.05$ was considered as statistically significant.

\section{Results}

\subsection{Demographic characteristics}

The recipients included 15 (50\%) males and 15 (50\%) females. The median age was 7 months (4-48 months), and the mean height and weight were $69.07 \pm 9.98 \mathrm{~cm}$ and $8.09 \pm 2.63 \mathrm{~kg}$. The mean body mass index (BMI) was $(16.78 \pm 2.67)\left(\mathrm{kg} / \mathrm{m}^{2}\right)$. The diagnosis was BA in 11 $(36.7 \%)$ patients and BA treated with Kasai surgery in $19(63.3 \%)$ patients. Table 1 summarizes the demographic information of the pediatric recipients.

\begin{tabular}{lcc}
\hline Characteristics & Mean (SD) & Median (range) \\
\hline \hline Age (years) & $10.13(9.53)$ & $7(4-48)$ \\
Height $(\mathrm{cm})$ & $69.07(9.98)$ & $66(58-100)$ \\
Weight $(\mathrm{kg})$ & $8.09(2.63)$ & $7.60(4.90-16.50)$ \\
BMl $\left(\mathrm{kg} / \mathrm{m}^{2}\right)$ & $16.78(2.67)$ & $16.20(13.70-26.70)$ \\
Weight Z score & $-0.89(1.53)$ & $-0.84(-4.21$ to 1.63$)$ \\
Height Z score & $-1.31(1.49)$ & $-1.11(-4.57$ to 1.48$)$ \\
BMl Z score & $-0.07(1.68)$ & $-0.61(-2.49$ to 5.20) \\
BMD & $0.12(0.86)$ & $0.10(-1.00$ to 1.90$)$ \\
Ca & $1.74(0.14)$ & $1.71(1.53-2.10)$ \\
Mg & $1.26(0.09)$ & $1.26(1.07-1.51)$ \\
Cu & $22.03(2.15)$ & $22.86(17.38-24.98)$ \\
Fe & $6.13(0.96)$ & $6.09(4.21-7.76)$ \\
Zn & $56.30(9.79)$ & $57.90(38.27-70.52)$ \\
Vitamin D & $8.09(8.09)$ & $5.40(1.93-36.42)$ \\
Vitamin D2 & $1.19(0.63)$ & $1.06(0.34-3.24)$ \\
Vitamin D3 & $6.92(8.54)$ & $3.20(1.01-35.80)$ \\
Vitamin D ratio & $0.42(0.39)$ & $0.31(0.02-1.62)$ \\
\hline
\end{tabular}

Table 1. Demographic characteristics of recipients.

Note: SD: standard deviation; BMI: body mass index; BMD: bone mineral density; Ca: calcium; Mg: magnesium; Cu: cuprum; Fe: iron; Zn: zinc. 


\subsection{Nutritional data}

The mean weight, height, and BMI $Z$ values were within the normal range ( -2 to 2$)$. The mean values of vitamin D and vitamin D3 were much lower than the normal range. The blood concentration of trace elements was close to normal. The content of iron was lower than the normal range, and calcium reached the baseline of the normal range.

\subsection{Data analysis of laboratory tests}

The data from Days 1, 2, 3, 4, 5, 6, 7, 14, 30, and 60 after liver transplantation were analyzed. The clinical outcomes are summarized in Table 2. WBC rose above the normal range at Day 6 , and neutrophil percentage (N\%) increased 7 percentage points at Day 2; both values declined to within the normal range at Day 30 . HB was almost always stable. PLT remained within the normal range. Total protein (TP) and ALB gradually normalized. ALT, AST, direct bilirubin (DB), and TB decreased and normalized from Day 14 onward, while alkaline phosphatase (AKP) and gamma-glutamyl transpeptidase (GGT) increased on Day 30. These indexes on Day 60 were also analyzed according to the gender, diagnosis, blood type, nutritional status, and graft-to-recipient weight ratio (GRWR), and the values are summarized in Table 3.

\subsection{Statistical analyses}

The liver function at Day 60 was also analyzed according to the gender, diagnosis, blood type, nutritional status, and GRWR, and Table 3 summarizes the results. The liver function did not show a significant difference in terms of the gender, blood type, diagnosis, and GRWR. However, the posttransplant ALT, GGT, and bile acid of the group who had not received the Kasai operation were higher than those of the group who had received the Kasai operation. The posttransplant ALT, AST, GGT, and bile acid of the group with GRWR lower than 1\% were higher than those of the group whose GRWR was higher than $1 \%$. We divided these recipients into two groups according to their weight $Z$ value, and the statistical analysis showed that the AST and glucose (Glu) at Day 60 were significantly different, with $P<0.05$.

\subsection{Correlation analysis}

We selected the liver function at Day 60 , weight $Z$ value, and the concentration of vitamin $D$ for the correlation analysis. The concentrations of vitamin $D$ and the AST showed a positive correlation relationship, with $P<0.05$ (Table 4).

\section{Discussion}

Liver transplantation has been a definite therapeutic treatment option for patients with end-stage liver disease. Liver grafts from cadaveric donors are not sufficiently available or capable of treating the waiting patients; therefore, LDLT is replacing cadaveric organ transplantations. ${ }^{7,8}$ Moreover, with the development of surgery skills, LDLT has become the most useful option to treat children with pediatric end-stage liver diseases such as congenital BA. However, few studies have evaluated the pre- and posttransplant nutritional status in children. Furthermore, the early detection of complications after transplantation is difficult. To aid in the detection of early complications, measurements of conventional liver markers, such as TB, AKP, GGT, and the transaminases (ALT and AST) are commonly used worldwide. ${ }^{9}$ In this study, we focused on the nutritional parameters and the data after the operation, especially the liver function. We hope to provide information from our experience to improve the future preoperative management and postoperative follow-up.

WBC, HB, and PLT generally increased and the N\% decreased after the surgery; this decrease was likely related to infection and immunosuppressive medicine. TP, ALB, ALT, AST, DB, and TB slowly normalized, while the AKP increased. It is reported that during the first 40 days, unexplainable troughs and peaks of increased AKP precede the increases in AST, ALT, and GGT as well as in bilirubin (at 20 days). ${ }^{9}$ This phenomenon could be related to possible biliary complications within the graft. Kim et al. ${ }^{10}$ reported that ALT, AST, TB, and GGT levels rapidly increased at 7 days after surgery compared to those before surgery and then decreased at 16 months after surgery compared to those before surgery. However, this study did not support these findings. The ALT, AST, TB, and GGT levels of this study slowly decreased and stabilized, and there was almost no fluctuation compared with that in the literature mentioned. When the liver is damaged by infection, bilirubin is reported to be increased ${ }^{11}$; therefore, special attention should be paid to liver function after surgery to help the recipients heal.

This study suggests that the ALT, AST, TB, and GGT levels decrease within 7 days after transplantation, which means that this period of time is very important. Table 2 shows that the creatinine was steady and that the PT increased slightly during the first 2 days and then decreased to the normal range. The renal function of LDLT pediatric recipients was relatively stable. Furthermore, the glycemia increased at Day 2 and then normalized. The relationships between intraoperative hyperglycemia and posttransplant outcomes have not 


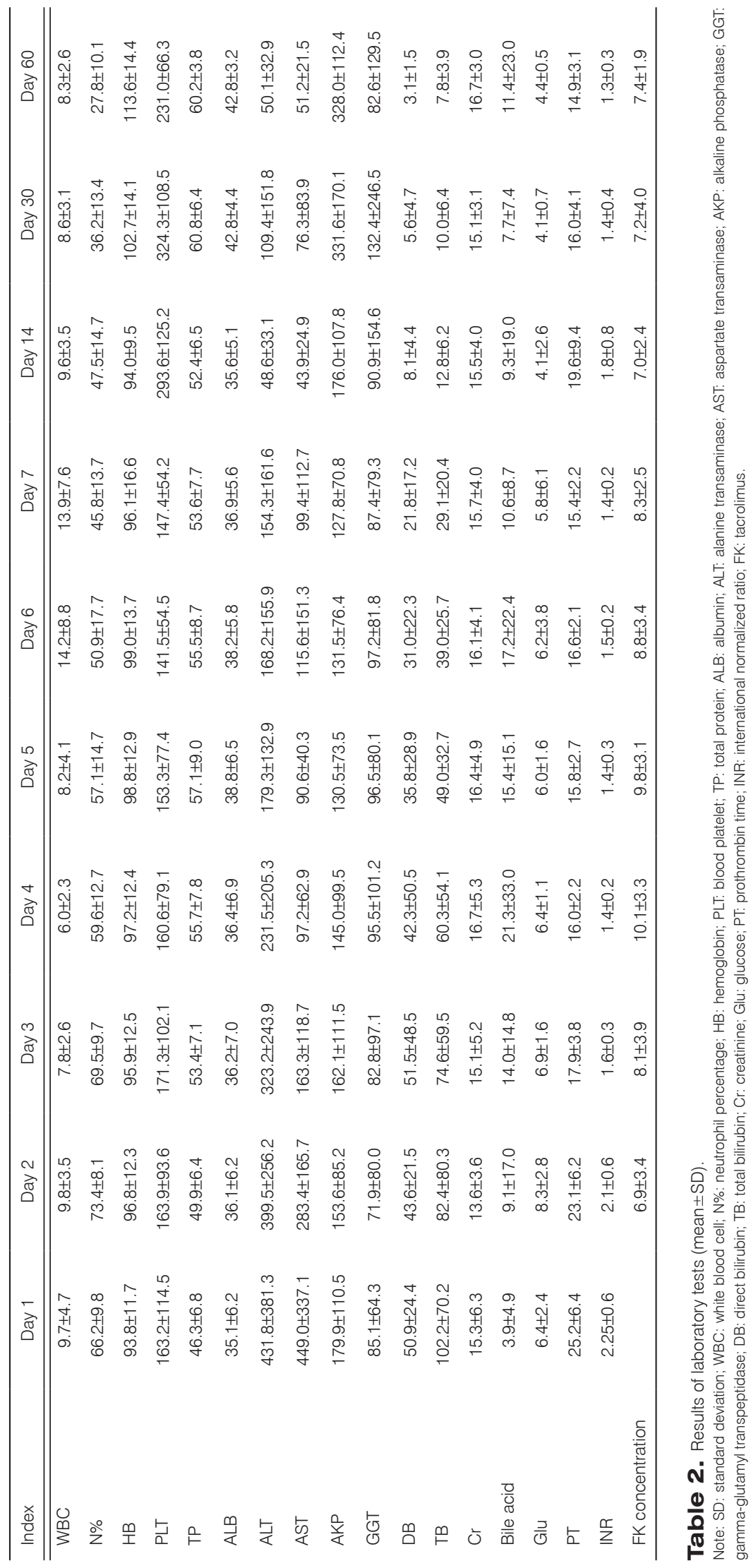




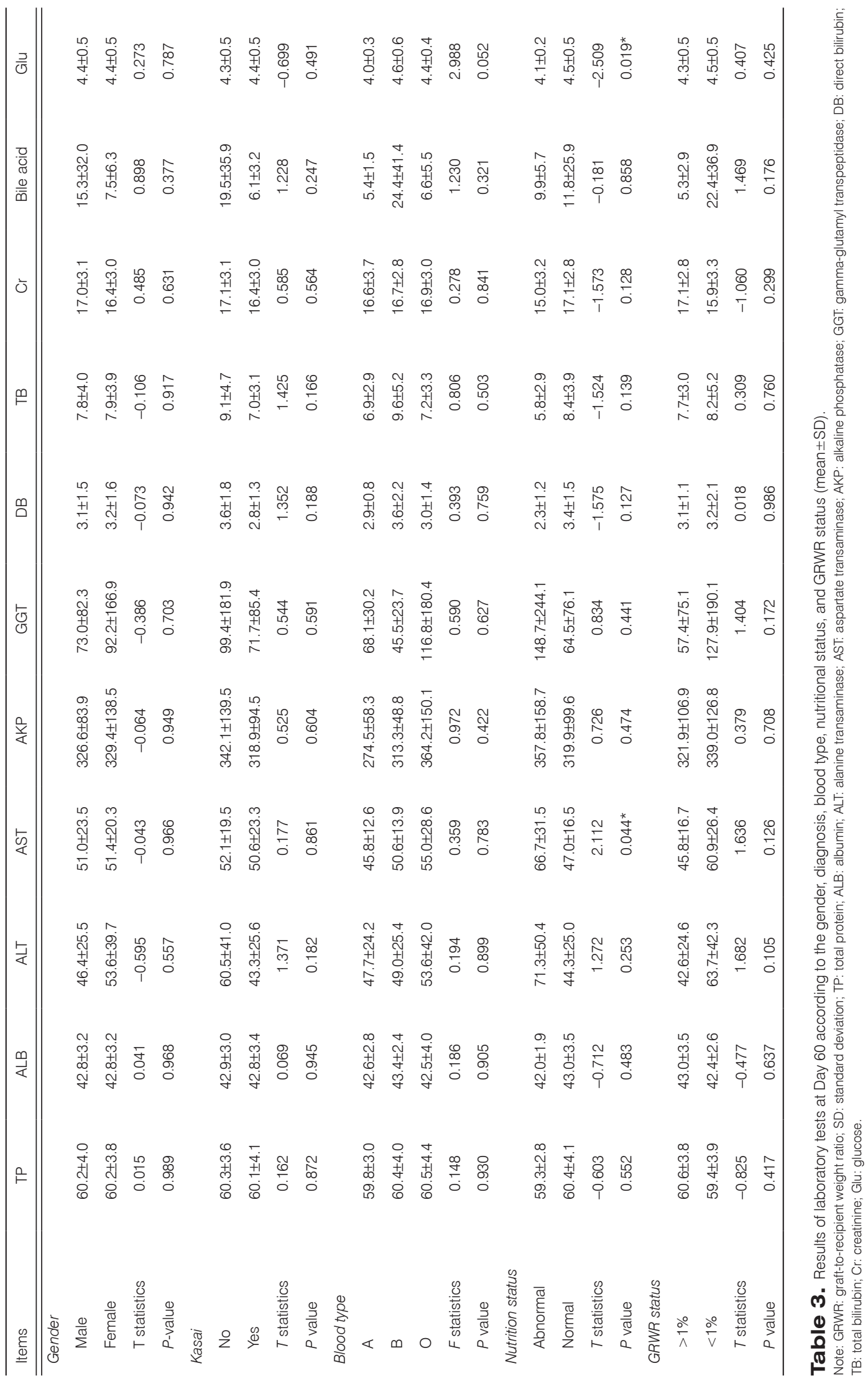




\begin{tabular}{|c|c|c|c|c|c|c|c|c|c|c|c|}
\hline Items & TP & ALB & ALT & AST & AKP & GGT & DB & TB & $\mathrm{Cr}$ & Bile acid & Glu \\
\hline \multicolumn{12}{|l|}{ Weight $Z$ value } \\
\hline Correlation & -0.062 & 0.054 & -0.349 & -0.288 & -0.171 & -0.281 & 0.189 & 0.196 & -0.121 & -0.011 & 0.345 \\
\hline$P$ value & 0.753 & 0.784 & 0.068 & 0.138 & 0.383 & 0.147 & 0.336 & 0.317 & 0.611 & 0.956 & 0.078 \\
\hline \multicolumn{12}{|l|}{ Vitamin D } \\
\hline Correlation & 0.201 & 0.120 & 0.197 & 0.458 & -0.049 & 0.034 & -0.115 & -0.036 & 0.318 & -0.203 & 0.029 \\
\hline$P$ value & 0.325 & 0.561 & 0.335 & $0.019 *$ & 0.811 & 0.868 & 0.575 & 0.862 & 0.113 & 0.321 & 0.892 \\
\hline
\end{tabular}

Table 4. Correlation between weight $Z$ value, Vitamin D level, and liver function.

Note: TP: total protein; ALB: albumin; ALT: alanine transaminase; AST: aspartate transaminase; AKP: alkaline phosphatase; GGT: gamma-glutamyl transpeptidase; DB: direct bilirubin; TB: total bilirubin; Cr: creatinine; Glu: glucose.

been widely studied. ${ }^{12,13}$ It has been reported that the glycemia increases abruptly after graft reperfusion ${ }^{14}$, and this initial increase in blood Glu is directly generated by Glu release from the donated graft. ${ }^{15}$ However, the glycemia in this study was relatively stable, which indicates that the grafts of the living donors were of higher quality than the DCD grafts. Table 2 also shows that the tacrolimus concentration and its standard deviation were relatively high during the first 7 days and then reached an acceptable level. Tacrolimus, an immunosuppressive drug, has a narrow therapeutic window and large pharmacokinetic variability with a poor correlation between the drug-dosing regimen and blood concentration. ${ }^{16}$ Therefore, more studies are necessary to decide on an ideal dose and medication strategy of tacrolimus.

Growth retardation is one of the most prominent consequences of childhood cholestatic liver diseases. ${ }^{17}$ The mean BMI in this study was 16.78 , which was lower than the standard, verifying the abovementioned conclusion. The pathogenesis of malnutrition is multifactorial and includes reduced calorie intake, fat malabsorption, abnormal protein metabolism, and increased energy expenditure. Therefore, the pretransplant nutritional evaluation and therapy appear to be very important. The current posttransplant pediatric patient survival rate exceeds $80 \%$, and achieving appropriate physical growth and development has become one of the longterm objectives. ${ }^{17}$ Determining how to conduct the nutritional follow-up effectively is necessary, in addition to the follow-up of liver function. Diminished bile flow leading to decreased intraluminal bile acid concentration appears to be the major pathogenic mechanism responsible for malnutrition and growth retardation. ${ }^{18}$ Consequently, we should advise patients to undergo a routine ultrasound examination to determine whether the bile duct is unobstructed. In addition to the height and weight, regular growth examination should also be performed to allow us to monitor the growth trend of these patients and help them grow normally.
The ALT, GGT, and bile acid of the group who did not have previous Kasai were higher than those of the group who had received the Kasai operation, which indicated that the latter group had a better prognosis, though the difference was not statistically significant. Prabhakran ${ }^{19}$ reported that liver transplantation must be preceded by the Kasai operation to provide the maximum benefits to patients. Despite the results of this study and the results in the literature, we should still be cautious about whether the Kasai procedure is useful in liver transplantation. More experimental research is needed to address this issue. However, if we think that we have the opportunity of a Kasai operation and ensure that there are no serious complications, we might create a favorable surgical condition, which is a necessary criterion for better outcomes after transplantation.

It is well recognized that poor nutritional status is associated with increased morbidity and mortality rates after liver transplantation. ${ }^{20,21}$ Impaired Glu tolerance is frequently observed in patients with cirrhosis. ${ }^{22,23}$ The statistical analysis showed that AST and Glu at Day 60 were significantly different according to the nutritional status, with $P<0.05$. Better nutritional status appeared to predict better liver function after liver transplantation. During the observation period, we found that those who have good nutritional status recovered much faster in terms of their liver function. Moreover, their length of hospital stay was also shorter. It was reported that perioperative nutritional support, including preoperative supplementation of branched chain amino acid (BCAA)enriched nutrients as a late evening snack, immunonutrition, and early enteral nutrition, significantly improved the overall survival after LTx in patients with low skeletal muscle mass. ${ }^{24}$ Further study is necessary to prove the benefits of nutritional intervention in the group of Chinese pediatric patients.

The ALT, AST, GGT, and bile acid of the group whose GRWR was lower than $1 \%$ were higher than those of the group whose GRWR was higher than $1 \%$, which sug- 
gested that the latter achieved better outcomes, though the difference was not statistically significant. It has been reported that the use of small-for-size grafts (less than 1\% of recipient body weight) leads to lower graft survival, and small-for-size graft syndrome can be avoided if GRWR is $>1 \% .^{25,26}$ The results of this study verified this viewpoint of the literature; we believe that when the GRWR is more than $1 \%$, the prognosis will be better. However, we should not narrow our point of view and should also consider the possibility of impaired venous outflow or portal hyperperfusion leading to graft failure. In other words, we should not only assure the appropriate GRWR but also monitor the flow and pressure of the vein or artery.

The correlation analysis showed that the concentration of vitamin $D$ and AST had a midrange positive correlation relationship, with $P<0.05$. In a low vitamin D population, high serum $25(\mathrm{OH})$ vitamin D concentrations were associated with a significantly lower risk of deaths due to CLD. ${ }^{27}$ Our results suggest a strong relationship between vitamin $\mathrm{D}$ concentrations and liver function. Vitamin $D$ had a protective role during the whole process of liver transplantation. Vitamin $D$ deficiency is highly prevalent in CLD patients, and vitamin $\mathrm{D}$ levels are inversely related to the severity of CLD. ${ }^{28}$ Therefore, it is necessary to perform some interventions before liver transplantation. We could test the level of vitamin $D$ in patients waiting for the operation and then give them the relevant treatment. In this way, we might reduce the risk of surgery and accelerate the recovery of the graft.

\section{References}

1. Dopazo C, Bilbao I, Castells LL, et al. Analysis of adult 20-year survivors after liver transplantation. Hepatol Int. 2015;9:461-470.

2. Zekri AR, Salama HM, Medhat E, et al. T The impact of repeated autologous infusion of haematopoietic stem cells in patients with liver insufficiency. Stem Cell Res Ther. 2015;6:118.

3. Zhou J, Shen Z, He Y, Zheng S, Fan J. The current status of pediatric liver transplantation in Mainland China. Pediatr Transplant. 2010;14:575-582.

4. García-Rodríguez MT, Piñón-Villar Mdel C, LópezCalviño B, et al. Assessment of nutritional status and health-related quality of life before and after liver transplantation. BMC Gastroenterol. 2015; $15: 6$.

5. Dasarathy S. Consilience in sarcopenia of cirrhosis. J Cachexia Sarcopenia Muscle. 2012;3:225-237.

6. Periyalwar P, Dasarathy S. Malnutrition in cirrhosis: Contribution and consequences of sarcopenia

\section{Conclusions}

From this study, we have increased the knowledge of the laboratory data, which could help to provide more information about the outcomes of the patient and the graft after transplantation. We also detected some influencing factors that we should pay attention to in clinical practice. In conclusion, better nutritional status predicts good outcomes after LDLT.

\section{Limitations}

The limitations of this study are that it is a nonrandomized, single-center, non-comparative study with a relatively short follow-up period. Further studies with control groups are necessary to strengthen our findings.

\section{Ethical approval}

The IRB approved the protocol.

\section{Conflicts of interest}

All contributing authors declare no conflicts of interest.

\section{Acknowledgment}

We thank Dr. Miriam Corcer who serves at the King's College Hospital, London, England, for helping us with the English writing.

on metabolic and clinical responses. Clin Liver Dis. 2012;16:95-131.

7. Ringe B, Pichlmayr R, Wittekind C, Tusch G. Surgical treatment of hepatocellular carcinoma: Experience with liver resection and transplantation in 198 patients. World J Surg. 1991;15:270-285.

8. Mazzaferro V, Regalia E, Doci R, et al. Liver transplantation for the treatment of small hepatocellular carcinomas in patients with cirrhosis. $N$ Engl $\mathrm{J} \mathrm{Med.}$ 1996;334:693-699.

9. Mercer D, Tang M, Marino IR, et al. Changes in biliary (high-molecular-mass) and liver isoforms of alkaline phosphatase after baboon-to-human liver transplantation. Clin Chem. 1994;40(7, Pt 1):1335-1339.

10. Kim MS, Lee HK, Kim SY, Cho JH. Analysis of the relationship between liver regeneration rate and blood levels. Pak J Med Sci. 2015;31:31-36.

11. Eason JD, Gonwa TA, Davis CL, Sung RS, Gerber $\mathrm{D}$, Bloom RD. Proceedings of consensus conference 
on Simultaneous Liver Kidney Transplantation (SLK). Am J Transplant. 2008;8:2243-2251.

12. Ammori JB, Sigakis M, Englesbe MJ, O'Reilly M, Pelletier SJ. Effect of intraoperative hyperglycemia during liver transplantation. J Surg Res. 2007;140:227-233.

13. Park C, Hsu C, Neelakanta G, et al. Severe intraoperative hyperglycemia is independently associated with surgical site infection after liver transplantation. Transplantation. 2009;87:1031-1036.

14. Chung HS, Kim ES, Rho MC, Park CS. Contribution of donor factors to post-reperfusion severe hyperglycemia in patients undergoing living donor liver transplantation. Ann Transplant. 2015;20:303-311.

15. Shangraw RE, Hexem JG. Glucose and potassium metabolic responses to insulin during liver transplantation. Liver Transpl Surg. 1996;2:443-454.

16. Gérard C, Stocco J, Hulin A, et al. Determination of the most influential sources of variability in tacrolimus trough blood concentrations in adult liver transplant recipients: A bottom-up approach. AAPS J. 2014;16:379-391.

17. Pawlowska J, Socha P, Jankowska I. Factors affecting catch-up growth after liver transplantation in children with cholestatic liver diseases. Ann Transplant. 2010;15:72-76.

18. Rodriguez-Baez N, Wayman K, Cox K.L. Growth and development in chronic liver disease. Neoreviews. 2001;2:e211-214.

19. Prabhakran K. Biliary atresia: Kasai and liver transplantation.AnnAcadMedSingapore. 1991;20:540-542.

20. Selberg O, Böttcher J, Tusch G, Pichlmayr R, Henkel E, Müller MJ. Identification of high- and low-risk patients before liver transplantation:
A prospective cohort study of nutritional and metabolic parameters in 150 patients. Hepatology. 1997;25:652-657.

21. Merli M, Giusto M, Gentili F, et al. Nutritional status: Its influence on the outcome of patients undergoing liver transplantation. Liver Int. 2010;30:208-214.

22. Petrides AS, Vogt C, Schulze-Berge D, Matthews D, Strohmeyer G. Pathogenesis of glucose intolerance and diabetes mellitus in cirrhosis. Hepatology. 1994;19:616-627.

23. Selberg O, Burchert W, vd Hoff J, et al. Insulin resistance in liver cirrhosis. Positron-emission tomography scan analysis of skeletal muscle glucose metabolism. J Clin Invest. 1993;91:1897-1902.

24. Kaido T, Ogawa K, Fujimoto $\mathrm{Y}$, et al. Impact of sarcopenia on survival in patients undergoing living donor liver transplantation. Am J Transplant. 2013;13:1549-1556.

25. Kiuchi T, Kasahara M, Uryuhara K, et al. Impact of graft size mismatching on graft prognosis in liver transplantation from living donors. Transplantation. 1999;67:321-327.

26. Chui AK, Rao AR, Island ER, Lau WY. Critical graft size and functional recovery in living donor

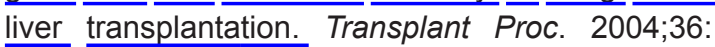
2277-2278.

27. Wang JB, Abnet CC, Chen W, et al. Association between serum $25(\mathrm{OH})$ vitamin $\mathrm{D}$, incident liver cancer and chronic liver disease mortality in the Linxian Nutrition Intervention Trials: A nested casecontrol study. Br J Cancer. 2013;109:1997-2004.

28. Stokes CS, Volmer DA, Grünhage F, Lammert F. Vitamin D in chronic liver disease. Liver Int. 2013;33:338-352.

How to cite this article: Lu YF, Wang Y, Huang MZ, et al. Preoperative nutrition and postoperative liver function: a correlation study of pediatric living donor liver transplantation. Front Nurs. 2018; 2: 157-164. https://doi. org/10.2478/fon-2018-0020 